Forthcoming in A. Sullivan (ed.), Sensations, Thoughts, Language: Essays in Honor of Brian Loar (Routledge)

\title{
Expression-Meaning and Vagueness
}

\author{
Stephen Schiffer
}

New York University

\begin{abstract}
Brian Loar attempted to provide the Gricean program of intention-based semantics with an account of expression-meaning. But the theory he presented, like virtually every other foundational semantic or meta-semantical theory, was an idealization that ignored vagueness. What would happen if we tried to devise theories that accommodated the vagueness of vague expressions? I offer arguments based on well-known features of vagueness that, if sound, show that neither Brian's nor any other extant theory could successfully make that adjustment, and this because, if sound, the arguments show not only that nothing can be the content of a vague expression, but also that no spoken language has a compositional semantics. This raises the question of what, really, are the facts about a language whose explanation might seem to require the language to have a compositional semantics, and whether there might not be a way to explain those facts on the assumption that the language doesn't have a compositional semantics. In response to this question I offer a rough sketch of a view designed to suggest how what needs to be explained might be explained without appeal to compositional semantics.
\end{abstract}

$* * *$

In "Two Theories of Meaning," the most important of his papers in the philosophy of language, Brian Loar asked:

What facts about a sentence constitute its meaning-that is, its meaning in abstraction from any particular utterance, and in so far as it belongs to the language of a particular population?

The answer he proceeded to give was a theory of expression-meaning in support of the Gricean program of intention-based semantics (IBS). IBS is a two-stage program for reducing all questions about the intentionality of speech acts and linguistic expressions to questions about the intentionality of thought: in the first stage a certain notion of speaker-meaning is to be taken as foundational in the theory of meaning and defined, without recourse to any public-language 
semantic notions, in terms of acting with certain audience-directed intentions, and in the second stage the semantic features of linguistic expressions are to be defined in terms of the now defined notion of speaker-meaning, together with certain ancillary notions, such as that of convention, also to be defined in terms of non-semantic propositional attitudes. A widely-held view about the relation between language and thought is that it is only our intentional mental states-believing, intending, and the like - that have original intentionality, intentionality that doesn't have its source in something else's intentionality; the intentionality of speech acts and words is derived intentionality, intentionality inherited from that of associated mental states. But that derivation story is plausible only if there is a plausible explanation of how speech acts and words inherit their intentionality from the propositional attitudes implicated in linguistic behavior. IBS aims to provide that plausible explanation. ${ }^{1}$ In the late sixties, when Brian began writing his D. Phil. thesis on Sentence Meaning, detailed revisions by Grice (1969) and myself (1972) of Grice's 1957 account of speaker-meaning were available to explain how propositional speech acts inherit their intentionality from the propositional attitudes in terms of which they, the speech acts, were defined, but there was nothing close to a comparably detailed account to show how expression-meaning was definable in terms of a Gricean account of speaker-meaning. The account of expressionmeaning Brian advanced in his dissertation was intended to fill that gap, and that is the account a revised version of which finally appeared in print in "Two Theories of Meaning." That account, in my estimation, is probably as good an account of expression-meaning as IBS can hope to achieve. But it shares a certain feature with every other IBS theory, and, as we'll see, that feature proves to be the banana peel under every IBS theorist's heel. The feature to which I allude is that every theory constructed under the IBS banner ignores vagueness, as though it simply didn't exist, and thus makes no attempt to accommodate the vagueness of vague expressions or vague acts of speaker-meaning, notwithstanding that virtually every sentence we utter and virtually every act of speaker-meaning is vague.

${ }^{1}$ Much of the appeal of IBS for Brian lay in what he saw as its place in reducing all intentional notions to nonintentional notions. Once IBS succeeded in reducing semantic intentionality to mental intentionality, the task, as Brian conceived it, was then to reduce mental intentionality, and thereby all intentionality, to functional notions that enjoy wholly physical realizations. The aim of his book Mind and Meaning (1981) was to fulfill that physicalistic ambition. 
Of course, proponents of IBS knew what they were doing. Part of the reason they ignored vagueness was that not to ignore it would require them to have a theory of vagueness, which they didn't have, and couldn't have had, since no IBS theorist had ever worked on vagueness. Yet that doesn't explain why IBS theorists developed their semantic theories under the pretense that there were no vague expressions or vague speech acts, for they might have concluded that, since virtually every expression and speech act was vague, it would be reckless to devise theories of meaning, reference, and speech acts when one had no idea how the vagueness of an expression affected its semantics or how the vagueness of an act of speaker-meaning affected what was meant in its performance. Evidently, however, Grice, Brian, and I must have believed that our ignoring vagueness was nothing worse than a useful idealization, akin, perhaps, to Galileo's ignoring friction in his theories of bodies in motion. Is that what we thought? Actually, I'm not sure any of us ever considered the question; at least I can't recall a single time in the countless conversations I had about IBS issues with Grice and Brian in the twenty years between 1965 and 1985 that the question even came up. At the same time, I suspect that if the question had been put to us we would have come up with some version of the harmless-idealization line. If that is right, then we were wrong: vagueness, as I will here try to show, defeats the program of intention-based semantics. Brian's account of expression-meaning makes it possible to sketch the architecture of a theory that realizes the IBS program, and once we have that we'll be positioned to see why vagueness defeats IBS. Now, in idealizing vagueness out of their theories IBS theorists were in good company, for virtually every presentation of a foundational semantic or meta-semantic theory ignores vagueness, and in seeing why vagueness defeats IBS we'll also see why it defeats pretty nearly every other theory of meaning.

\section{The Architecture of IBS}

That architecture has the following parts.

a. The most foundational IBS notion is that of speaker-meaning; this is where the semantic makes its first contact with the psychological. Speaker-meaning is the most general kind of illocutionary act, the genus of which all other kinds of illocutionary acts are species (see Schiffer 1972: Ch. IV). There are interrogative and imperatival acts of speaker-meaning, but present purposes will be adequately served if we identify speaker-meaning with assertoric speakermeaning (henceforth simply speaker-meaning), i.e. meaning that such-and-such is the case, as 
when in uttering 'J'ai faim' Odile meant that she was hungry. IBS takes speaker-meaning to be a relation, $S$ meant $p$, between a person $S$ and a proposition $p$ that she meant, where a proposition is any abstract entity that has truth conditions, has those truth conditions necessarily, and is mindand language-independent in that it belongs to no language and wasn't created by what anyone said or thought. ${ }^{2}$ That relation, as already noted, is then defined wholly in terms of acting with certain audience-directed intentions. There is no official IBS definition of speaker-meaning, and IBS theorists may disagree among themselves about how exactly the details of that definition should go, but the following toy conditions will highlight the features of an IBS account of speakermeaning most relevant to this essay:

For any person $S$, proposition $p$, and utterance $x,{ }^{3} S$ meant $p$ in uttering $x$ iff for some person $A$ and feature $\varphi, S$ intended it to be mutual (or common) knowledge between $A$ and $S$ that $x$ has $\varphi$ and, on the basis primarily of that, mutual knowledge that $S$ uttered $x$ intending her utterance of $x$ to result in $A$ 's actively believing $p$.

Let's call the value of ' $\varphi$ ' in an act of speaker-meaning its inference-base feature (its IB-feature, for short). Acts of speaker-meaning are typically performed by uttering sentences of a language common to the speaker and her audience, and, according to IBS, the IB-features of those sentences are their meanings. If you utter 'It's snowing' to communicate that it's snowing, the IB-feature of the sentence 'It's snowing' on which you rely is the meaning of that sentence in English. The reason IB-features are typically meaning properties is that meaning properties are optimal IBfeatures: if you want to tell your child that it's snowing, you would do much better to utter 'It's snowing' than to attempt to communicate that it's snowing by impersonating a snowflake. At the

2 As we will later see (in §IV), for present purposes it doesn't matter to which kind of propositionFregean, Russellian, functions from possible worlds into truth-values, whatever-the propositions we mean are taken to belong, provided they can be assigned in a compositional truththeoretic semantics, but for simplicity of exposition I will sometimes write as though they are taken to be Russellian propositions, i.e. structured entities whose basic components are the objects and properties our speech acts are about.

${ }^{3}$ Here, following Grice (1957), I use 'utterance' and its cognates as a technical term that applies both to linguistic and non-linguistic items and behavior. 
same time, a sine qua non of the IBS account of speaker-meaning is that the only intentional notions mentioned on its right-hand side are ordinary propositional-attitude notions, and, consequently, it's not a necessary condition for a person's meaning a proposition that what she utters have any sort of semantic property as its IB-feature. For example, during a lecture one might communicate to one's friend that one is bored by closing one's eyes and pretending to snore. The fact that the IB-feature needn't be a semantic feature is (supposed to be) what makes it in principle possible to define expression-meaning in terms of speaker-meaning. The IBS strategy for defining expression-meaning is to define it in terms of certain conventional regularities in communicative behavior that explain why a sentence's having the meaning it has in a certain population of speakers makes uttering that sentence an optimal way for a member of the population to communicate to another of its members a proposition that conforms to that meaning.

b. Having defined speaker-meaning, the next thing on the IBS agenda is defining all other agent-semantic notions in terms of the now defined notion of speaker-meaning. Here the most important notions are illocutionary act and speaker-reference. IBS dispatches illocutionary acts simply by identifying each kind of illocutionary act with a particular kind of act of speakermeaning (see Schiffer 1972, Ch. IV). As for speaker-reference, there are a few interrelated notions of speaker-reference, but the two most important for present purposes are notions of what I will call primary (or first-order) speaker-reference (Schiffer 1981; see also Neale 2016 and Schiffer 2016). I'll call the first of these notions referring-in (referring ${ }_{i}$ ) and define it thus:

$S$ referred $_{i}$ to $o$ in (the course of) uttering $x$ iff, for some $o$-dependent proposition $p_{o}, S$ meant $p_{o}$ in uttering $x .^{4}$

For example,

In nodding her head in response to the question "Is Henry coming to the party?", Jane meant the Henry-dependent proposition that Henry was coming to the party, and thus referred to Henry in nodding her head.

${ }^{4}$ An $o$-dependent proposition is one that is individuated partly in terms of $o$ and wouldn't exist if $o$ didn't exist. 
In uttering 'He's coming to the party' in response to the question "Is Henry coming to the party?", Jane meant the Henry-dependent proposition that Henry was coming to the party, and thus referred $\mathrm{i}_{\mathrm{i}}$ to Henry in (the course of) uttering that sentence.

Although in both examples Jane referred to Henry, there is an important difference between them: in the first example there was no part of Jane's "utterance" (in this case, her nodding) with which she referred to him, but in the second example there was; there she referred to him with the demonstrative pronoun 'he'. (And, we may add, it's because she referred to Henry with 'he' that the token of 'he' she uttered itself refers to Henry.) Referring-with (referring $w_{w}$ ) is the second of the two notions of primary reference to which I alluded, and it may be defined (at least to a first approximation) thus:

S referred ${ }_{w}$ to $o$ with expression $\varepsilon$ iff for some sentence $\Sigma$ containing $\varepsilon$, property $\varphi$ of $\varepsilon$, and audience $A, S$ uttered $\Sigma(\varepsilon)$ intending it to be mutual knowledge between $S$ and $A$ that $\varepsilon$ has $\varphi$ and, on the basis primarily of that, mutual knowledge that $S$ referred to $o$ in uttering $\Sigma(\varepsilon)$.

Primary speaker-reference, then, is de re speaker-meaning. To refer to a thing $o$ in an act of primary speaker-reference is to mean some proposition about o. In $(n>1)^{\text {th }}$ order speaker reference the speaker refers to a thing not in order to mean something about it, but in order to refer to a thing to which she is making an $(n-1)^{\text {th }}$ order reference. For example, in uttering

That boy next to Mary is noisy

the speaker is making a primary reference to that boy next to Mary and a secondary reference to Mary; and in

That boy next to that man talking to Mary is noisy

the speaker is making a primary reference to that boy next to that man talking to Mary, a secondary reference to that man talking to Mary, and a tertiary reference to Mary. And so on. ${ }^{5}$

c. Having defined speaker-meaning and all other agent-semantic notions in terms of nonsemantic propositional attitudes, the one remaining thing on the IBS agenda is to define the

${ }^{5}$ Schiffer (1981) contains a recursive definition of speaker-reference devised by Brian and myself which accommodates speaker-reference at any order. 
semantic properties of public-language expressions in terms of the already constructed IBS definitions. The strategy for doing this that best suited IBS, and the one Brian adopted, was inspired by David Lewis (1969). That strategy has two parts. In the first part, a language is taken to be a certain kind of abstract object that may or may not be used by anyone - namely, a finitely specifiable function from infinitely many finite strings of sounds (marks, gestures, whatever) to things of the kind meanings must be. If $L$ is such a function and $L(\varepsilon)=\mu$, then we may say that $\varepsilon$ means $\mu$ in $L$, but that stipulated use-independent notion of meaning is merely an expository convenience, and doesn't express the use-dependent notion of meaning that philosophers have long sought to understand, that is to say, the notion of meaning philosophers have in mind when they say that the meaning of a word is determined by its use. Now, if a language $L$ is the public language of a population $P$, the language in which members of $P$ communicate with one another, then every expression of $L$ will mean in $P$ what it means in $L$. Consequently, if we can say what must be the case in order for a language to be the language of a population, then we will have an account of what it is for $\varepsilon$ to mean $\mu$ in $P$, for any expression $\varepsilon$, meaning $\mu$, and population $P$. And since whether or not a language is the language of a given population will depend on how members of the population use the language, the account will answer the question of how meaning depends on use. Let's say that the public-language relation is that relation that must hold between a population $P$ and language $L$ in order for $L$ to be a public language of $P$. So, the second part of the Lewis-inspired strategy is to define the public-language relation, and for the IBS theorist this will require defining that relation in terms of the way members of $P$ use $L$ to perform acts of speakermeaning.

In order to implement this strategy the IBS theorist must first do two things. So far I've characterized IBS as holding that speaker-meaning is a relation between speakers and propositions of some stripe or other. The first thing the IBS theorist must do is settle on the stripe of the propositions we mean; this is essential in order to know what word meanings should be taken to be. IBS theorists may disagree among themselves as to the nature of the propositions that comprise the range of the speaker-meaning relation, but for convenience of exposition I will initially suppose them to be Russellian, structured entities made up of the objects and properties our speech acts are about. I don't really think they can be Russellian propositions, for I don't think that what a speaker would mean in uttering ' $\mathrm{J}$. K. Rowling is the author of the Harry Potter novels' is the same as what he would mean in uttering 'Robert Galbraith is the author of the Harry Potter novels', but once I 
reach the conclusions I need to reach relative to the Russellian assumption, it will be easy to show (in $\S I V$ ) that those conclusions would also have been reached relative to any other assumption about the nature of the propositions we mean. The second thing the IBS theorist must do is say what expression-meanings must be, relative to the assumption that the things we mean are Russellian propositions. IBS takes sentences to be devices for performing acts of speakermeaning, and it takes their meanings to provide bases from which hearers may infer what speakers mean in producing unembedded utterances of those sentences. If declarative sentences were context-insensitive, the IBS theorist could take their meanings to be the propositions that, all else being equal, speakers would mean in producing unembedded utterances of them, and it would take the meanings of words and other subsentential expressions to be the objects and properties that compose those propositions. But since hardly any of the sentences we utter are context-insensitive, IBS will want to take expression-meanings to be things that constrain what speakers can mean in uttering those sentences. This may be spelled out in the following way.

Like virtually every other philosopher who has written about context-sensitive expressions, IBS theorists recognize two kinds of meanings, which I'll call meanings and contents. Meanings are properties of expression-types, contents of expression-tokens. The meaning of an expressiontype $\varepsilon$ determines, for any $x$ and any token $\varepsilon_{\tau}$ of $\varepsilon$, what is required for $x$ to be the content of $\varepsilon_{\tau}$. Contents of declarative sentence tokens are (we're supposing) Russellian propositions, and if a proposition is the content of an unembedded token of a declarative sentence, then that proposition is what the speaker said in producing the token, if she said anything in producing it. The content of every expression token is its contribution to the content of the unembedded sentence token in which it occurs (an unembedded sentence token counts as occurring in itself). The meaning of a context-insensitive expression will assign the same content to every token of the expression which has a content, whereas the meaning of a context-sensitive expression will permit different tokens of the expression to have different contents. I'll call this way of understanding meanings and contents the meaning/content model (the M/C model, for short).

Given the M/C model it's useful to represent the meaning of an expression as a function from its tokens to their contents; to define such a function is to specify, for any possible content $c$ and expression $\varepsilon$, the conditions that must be satisfied in order for $c$ to be the content of a token 
of $\varepsilon{ }^{6}$ So conceptualized, the meaning of a context-insensitive expression will be a constant function that maps every token of the expression onto the same content, and the meaning of a

${ }^{6}$ On David Kaplan's (1989a, 1989b) version of the M/C model, meaning is called character, and is represented as a function from "contexts of utterance" to Russellian contents, where a "context of utterance" is a sequence of exactly the things needed to determine the content of any contextsensitive expression relative to such a "context." But: (i) the representation of an expression's meaning as a mapping of the expression's tokens onto their contents is merely a notational variant of Kaplan's characters if there really are sequences of things capable of determining the contents of context-sensitive expressions, since in that case any function from tokens to contents would have to be defined in terms of the way Kaplan's sequences determine the contents expressions have relative to them. (ii) It can't be assumed that there are Kaplanian sequences to determine the content of any context-sensitive expression, so Kaplan's representation of expression meanings begs questions that aren't begged by my construal of how the meaning/content model should represent meanings. (iii) It's arguable that for all but a few indexical expressions-e.g. 'I' and 'yesterday' (but not 'here' and 'now') - most of the work in determining the reference of a speaker's utterance of a demonstrative or indexical is done by the speaker's referential intentions (e.g. it's very arguable that in a speaker's utterance of 'He doesn't love her', the referent of 'he' is the male to whom the speaker referred with 'he' and the referent of 'her' is the female to whom the speaker referred with 'her'), and this, in fact, is the view Kaplan came to accept in (1989b). But then there is no need for a technical conception of "contexts of utterances" as Kaplanian sequences if the references of context-sensitive terms are determined by speakers' referential intentions (see Schiffer 2003, §3.7). Kaplan does offer an argument (1989a: §XIII) to show that we won't be able to have a logic capable of accounting for the validity of arguments formulated with sentences containing demonstratives or indexicals if we represent those arguments as containing utterances/tokens of those expressions, but the soundness of Kaplan's argument is discussable. It's also worth remarking that the practice among formal semanticists of representing referential pronouns and demonstratives as free variables and then giving the truth conditions for the sentences containing them relative to "contextually-determined assignment functions" isn't incompatible with taking the meaning of an expression to be a function from its tokens to their contents. 
context-sensitive expression will be a non-constant function whose values may differ from one token of the expression to the next. Since IBS takes a language to be a finitely specifiable function that maps its expressions onto their meanings, it is committed to taking the meaning of a complex expression to be a syntax-determined function of the meanings of its constituent words (or other morphemes); consequently, the only meanings that must be specified in defining a language are those given in the base-clauses for the language's morphemes. Specifying the meaning of a context-insensitive word merely requires saying what unique content each of its tokens must have. For example, the theorist might say that the meaning of 'Saul Kripke' is that function $f$ such that, for any token $\tau$ of 'Saul Kripke', $f(\tau)=$ Saul Kripke. As for context-sensitive words it will prove useful to see specifications of the functions that are their meanings as completions of the schema

For any token $\varpi_{\tau}$ of $\varpi$ and any $\mathrm{x}, f\left(\varpi_{\tau}\right)=x$ iff

(1) [eligibility condition] ...;

(2) [selection condition] ...,

where the eligibility condition for an expression specifies the condition something must satisfy in order for it to be eligible to be the content of a token of the expression, while the selection condition selects which of the things eligible to be the content is the content. For example, one might specify the meaning of the demonstrative 'she' thus:

$x$ is the content of a token $\tau$ of 'she' iff

(1) [eligibility condition] $x$ is female;

(2) [selection condition] $x$ is uniquely such that the speaker who produced $\tau$ referred to $x$ with $\tau$.

The schema admits the possibility of two kinds of limiting cases. At one limit, the eligibility condition is such that only one thing can satisfy it, and in this case the eligibility condition also does the work of the selection condition. The meaning of ' $I$ ' is like this if the content of a token of ' $I$ ' is the speaker who produced it. At the other limit, the eligibility condition imposes no restriction on the kind of thing that can be a content, and in this case the selection condition also does the work of the eligibility condition. Perhaps no word is at this limit, although 'it', when 
used as a demonstrative pronoun, would be if all it takes for a thing to be the content of a token of 'it' is that the speaker referred to it with the token. ${ }^{7}$

Having defined a language as a finitely specifiable mapping of sounds onto meanings, the IBS theorist sets out to complete his account of expression-meaning by defining the publiclanguage relation, the relation that must obtain between a population $P$ and a language $L$ in order for $L$ to be a public language of $P$. There were known to be problems that arose in attempting to define the public-language relation, perhaps the biggest being what David Lewis (1975) dubbed the meaning-without-use problem, and they were the problems Brian sought to solve in "Two Theories of Meaning." Now, in order for a definition of the public-language relation to provide an IBS account of expression-meaning its defining conditions must involve no ineliminable semantic notions, but only those propositional-attitude notions that, according to IBS, define speaker-meaning and convention, and elsewhere (2017) I have argued that that requirement can't be met, and that therefore there can be no correct IBS account of expression-meaning. Be that as it may however. I will now present a new argument to show why no IBS account of expressionmeaning can be correct. This argument will have relevance well beyond IBS, for, if it's sound, it will show that no account of expression-meaning that presupposes the $\mathrm{M} / \mathrm{C}$ model can be correct, and a corollary of this will be that natural languages don't have the kind of compositional semantics that nearly every philosopher of language and semanticist supposes they must have.

\section{Penumbral Shift and Context-Sensitivity}

How might the $\mathrm{M} / \mathrm{C}$ model accommodate vague terms when it's not ignoring their vagueness? Many vague expressions are obviously context-sensitive, whether or not their vagueness is taken into account, and therefore can be accommodated in the model only if it can assign them meanings that are specifiable by instances of the schema:

For any token $\varpi_{\tau}$ of $\varpi$ and any $\mathrm{x}, f\left(\varpi_{\tau}\right)=x$ iff

(1) [eligibility condition] ...;

(2) $[$ selection condition) $] \ldots$.

${ }^{7}$ I realize that my gloss of the labels eligibility condition and selection condition isn't exactly precise, but I don't think it needs to be more precise to be useful in the informal intuitive way I intend the labels to be useful. 
Such terms include, for example, 'here', 'fast', 'tall', and 'intelligent'. But other vague expressions will be treated by the model as context-insensitive when their vagueness is ignored. Such terms include 'boy', 'bachelor', 'bald man', 'alcoholic', 'logician', 'athlete', and 'triangular'. For example, when its vagueness is being ignored (and the things we mean are taken to be Russellian propositions), the IBS theorist would say that the meaning of 'boy' is a constant function that maps every token of the word onto the property of being a boy. Let's call such terms predicates*. What can the model say about predicates* when it's not ignoring their vagueness? Let's start by noticing that every predicate* has a feature, consequent on its being vague, such that, owing to its having that feature, the predicate* can be accommodated in the $\mathrm{M} / \mathrm{C}$ model only if it can be assigned a context-sensitive meaning, i.e. a meaning that is specifiable as an instance of the just displayed schema. I call this feature penumbral shift, and it may be explained in the following way.

The notion of penumbral shift is defined in terms of the notion a penumbral profile. Every token of every predicate* has a penumbral profile, where two predicate* tokens have the same penumbral profile just in case if either applies to a thing, then so does the other; if either is such that it's indeterminate whether it applies to a thing, then so is the other; if either is such that it's indeterminate whether it's indeterminate whether it applies to a thing, then so is the other; and so on ad infinitum. ${ }^{8}$ Penumbral shift is then the fact that the penumbral profiles of a predicate*'s

${ }^{8}$ My use of 'vague', 'determinate', 'indeterminate', and 'borderline' throughout this essay is pretheoretic in that nothing I say will rely on any particular philosophical theory of vagueness but will instead remain neutral on that score. So, for example, as far as this essay is concerned it's not assumed that 'It's indeterminate whether Harold is bald' entails 'It's neither true nor false that Harold is bald'. I will, however, assume that: (i) ' $x$ is borderline $F$ ' entails 'It's indeterminate whether $x$ is $F$ '. (ii) 'It's indeterminate whether $x$ is $F$ ' doesn't entail ' $x$ is borderline $F$ '. For example, if, as many suppose, it's indeterminate whether the continuum hypothesis is true, that wouldn't entail that it was borderline true or even vague. In this essay, however, the only indeterminacy that will be at issue is vagueness-induced indeterminacy, so far all that presently matters 'It's indeterminate whether $x$ is $F$ ' is equivalent to ' $x$ is borderline $F$ '. (iii) If it's indeterminate whether $\mathrm{x}$ is $\mathrm{F}$, then it's impossible for anyone to know whether $x$ is $F$. If, for example, it's indeterminate whether Harold is bald, then nothing can count as one's discovering 
tokens may shift somewhat from one token to the next; that is to say, two tokens of a predicate* may have somewhat different penumbral profiles. The "somewhat" qualification is important. For example, if Clyde is a man whose scalp is as hairless as a billiard ball and on whose scalp no hair can grow, then every token of 'bald man' must be true of Clyde, and if Clyde is blessed with a head of hair like the one Tom Cruise appears to have, then every token of 'bald man' must be false of him. At the same time, penumbral shift makes it possible for there to be three tokens of any predicate*, one of which is true of the thing to which it's applied, another of which is false of the thing to which it's applied, and still another of which is such that it's indeterminate whether it's true of the thing to which it's applied. Here are three examples:

- At a party George is asked whether Henrietta came to the party with anyone, and he replies 'She came with some bald guy'. That utterance would most likely be accepted in the context in which it occurred as true, even if it transpires that the man in question shaves his scalp but wouldn't be said to be bald if he let his hair grow out.

that Harold is in fact bald or that he is in fact not bald. If one did come to know that Harold was bald, then one would thereby come to know that it's not indeterminate whether Harold is bald. (iv) One might wonder what the difference is supposed to be between $x$ 's being $F$ and $x$ 's being determinately $F$. What, for example, is the difference between its being true that it's raining and its being determinately true that it's raining? If they are two distinct facts, in what can the difference between them consist? Are we to imagine that it's raining harder if we suppose that it's determinately true, as opposed to being merely true, that it's raining? Of course not. It's not that one might know that Sadie is lethargic and then wonder whether she's also determinately lethargic. To understand my vagueness-related use of the jargon expressions 'determinate' and 'indeterminate' it's enough to know that, while I assume that ' $x$ is determinately $F$ ' entails ' $x$ is $F$ ', I don't assume that ' $x$ is $F$ ' doesn't entail ' $x$ is determinately $F$ '. For ' $x$ is not determinately $F$ ' must be consistent with 'It's indeterminate whether $x$ is $F$ ', and we don't want to assume that to entail ' $x$ is not $F$ '. So we can't deny that it's indeterminate whether $x$ is $F$ by claiming that $x$ is $F$ or that $x$ isn't $F$, but we can deny it by claiming that $x$ is determinately $F$ or that $x$ is determinately not $F$. The usefulness of ' $x$ is determinately $F / \operatorname{not} F$ ' resides in its incompatibility with 'It's indeterminate whether $x$ is $F$ '. 
In another conversation, however, in which the discussion is about hereditary baldness, someone might correct a remark about the same man by saying 'No; he's not bald; he just shaves his scalp', and that utterance, in that context, would very likely count as true. In still another context the question is raised whether a man who shaved his scalp would be bald if no one would take him to be bald if he stopped shaving his scalp and let his hair grow out, and in that context it might be true to say "That's undetermined by the use of "bald" in everyday speech; such a man would be neither determinately bald nor determinately not bald'.

- In a community in which people typically marry before the age of twenty, an utterance of 'He's a bachelor' may count as true when said of an unmarried eighteen-year-old male, whereas in a conversation among New Yorkers, where for both men and women the average age for a first marriage is between thirty and thirty-five, an utterance of 'He's a bachelor' would most likely not count as determinately true when said of an unmarried eighteen-year-old male, and may even count as false.

- An utterance of 'Mary is getting married to a boy from Boston' would count as false if the male whom she is about to marry is fiftytwo years old, but is apt not to count as false if he is twenty-seven years old. At the same time, if every one of the seven male professors in one's department is over forty except Henry, who is twenty-seven, an utterance of 'Six men and one boy are professors in my department' would count as a misuse of 'boy'.

Let's take 'boy' as our exemplar of a predicate* and ask how a theorist might attempt to accommodate it in the $\mathrm{M} / \mathrm{C}$ model when its vagueness is taken into account.

We may start by asking what the IBS theorist might say about the content of the token of 'boy' uttered by Jack in the following example.

Jack and Jill are on a first date, and at the moment are telling one another about their respective families. Jack has just told Jill that he has a sister, 
Jane, who is the mother of a seven-year-old child named 'Morgan', and then, realizing that 'Morgan' is a unisex name, adds 'Morgan is a boy'.

IBS holds that the things we mean, and thus the contents of declarative sentence tokens, are propositions, and we are provisionally assuming that those propositions are Russellian propositions. If an IBS theorist were ignoring vagueness, she would say that what Jack meant in uttering 'Morgan is a boy', and the content of the token of that sentence he uttered, is the singular proposition $<$ Morgan, the property of being a boy $>;^{9}$ for if the theorist is ignoring vagueness, then she would say that 'boy' is context-insensitive, and that its meaning is that function $f$ such that for any token $\tau$ of 'boy' and any $x, f(\tau)=x$ iff $x=$ the property of being a boy, and that therefore the content of the token of 'boy' Jack uttered is the same as every other token of 'boy', viz. the property of being a boy. But now we are supposing her not to be ignoring vagueness, and 'boy' is vague. It's vague because even if the token of 'Morgan is a boy' Jack uttered is determinately true or determinately false, it might have been neither: Morgan might have been a borderline case of a thing to which 'boy' applies, either because Morgan is a borderline case of a male, or of a human being, or because he's of an age such that it's indeterminate whether 'boy', as uttered by Jack, applies to a human male of that age. So what can the theorist say about the content of the token of 'boy' Jack uttered? All that we can say at this point is that its content can't be the property of being a boy, and this because there can be no such property as the property of being a boy. For if there were such a property, then it would be the content of every token of 'boy', and penumbral shift secures that no property is the content of every token of the word. Because of penumbral shift, the M/C model must treat 'boy' as a context-sensitive term; that is to say, if the model is to account for the word's meaning in a way that takes account of its vagueness, then it can do so only by finding a true completion of the schema:

For any token $\tau$ of 'boy' and any property $\varphi, f(\tau)=\varphi$ iff

(1) [eligibility condition] ...;

(2) [selection condition] ....

What options does the $\mathrm{M} / \mathrm{C}$ model have for achieving such a completion? In order to answer this, we would first have to know what options are available to the model for saying what the contents

${ }^{9}$ For convenience of exposition I will ignore syntax-determined temporal reference when nothing turns on it. 
of tokens of 'boy' are, given the provisional assumption that the contents of declarative sentence tokens are Russellian propositions. We can make this question more tractable by returning to the example of Jack's utterance of 'Morgan is a boy' (above p. ...) and asking what the M/C model can say about the content of the token of 'boy' Jack uttered. However, since properties, like propositions, are mind- and language-independent entities, the $\mathrm{M} / \mathrm{C}$ theorist can't say what the content of a token of 'boy' might be without first taking a stand on the issue of ontic vagueness, or vagueness in the world.

\section{Ontic Vagueness and the Meaning of 'Boy'}

The issue of ontic vagueness is a contest between a view I'll call no-vagueness-in-the-world and one I'll call vagueness-in-the-world. What exactly is at issue in this contest doesn't escape vagueness, but to what I trust is a good-enough approximation we may say that no-vagueness-inthe-world holds that nothing outside of language and thought can be vague in its own right (i.e. independently of the vagueness of language and thought), while vagueness-in-the-world holds that properties and things outside of language and thought may be vague in their own right. As a terminological convenience I'll say that things that aren't vague in their own right are metaphysically precise, and that things that are vague in their own right are metaphysically vague. A proposition is metaphysically precise provided it's necessarily the case that there is a fact of the matter as to what truth-value, if any, it has. If bivalence holds for propositions, then a proposition is metaphysically precise provided it's necessarily the case that it's a fact that it's true or else a fact that it's false. If, as Frege held, presupposition failure can deprive a proposition of a truthvalue, then a proposition is metaphysically precise provided it's necessarily the case that it's a fact that it's true, a fact that it's false, or a fact that it's neither true nor false. And if there are three or more truth-values, then a proposition is metaphysically precise provided it's necessarily the case that there is a fact of the matter as to which truth-value it has. For convenience of exposition I will assume that metaphysically-precise propositions are necessarily bivalent, either true or else false, and, as a matter of terminology, I'll also say that it can't be metaphysically indeterminate what its truth-value is, but of course that won't preclude its being indeterminate what its truth-value is in an epistemological sense of 'indeterminate'. Given the assumption of bivalence, we can also say that a property is metaphysically precise provided every metaphysically-precise thing is such that, necessarily, it's a fact that it has the property or else a fact that it doesn't have it. The restriction 
to metaphysically-precise things is to allow for the possibility of a metaphysically vague object's being such that, owing to its vagueness, it's metaphysically indeterminate whether a particular metaphysically-precise property applies to it. Henceforth, in the interest of readability, 'metaphysically-' will mostly be invisible in occurrences of 'metaphysically-precise' and 'metaphysically-vague'.

We can now say that, subject to a certain qualification, if 'boy' can be accommodated in the $\mathrm{M} / \mathrm{C}$ model relative to the assumption that Russellian propositions are the contents of declarative sentence tokens, then the following options (in which $\beta$ is the token of 'boy' Jack uttered) are the ones worth taking seriously:

(A) Some precise property is the content of $\beta$.

(B) No precise property is such that it's determinately the content of $\beta$, but myriad precise properties are each such that it's indeterminate whether it's the content of $\beta$.

(C) Some vague property is the content of $\beta$.

(D) No vague property is such that it's determinately the content of $\beta$, but myriad vague properties are each such that it's indeterminate whether it's the content of $\beta$.

Although (A)-(D) are the only answers we need to consider, logical space contains infinitely more possible answers. In fact, for every answer $X$ contained in logical space, logical space also contains the answer that it's indeterminate whether $X$, so that, for example, in addition to (A), logical space also contains the answers:

(A2) It's indeterminate whether (A).

(A3) It's indeterminate whether (A2).

(A4) It's indeterminate whether (A3)

But once our critical examination of (A)-(D) is concluded it will be clear why we have no need to consider permutations of them induced by higher-order vagueness. ${ }^{10}$

${ }^{10}$ Another option in logical space not worth taking seriously is that 'boy' has a constant meaning that changes from moment to moment. 
Now that we know the options available to the $\mathrm{M} / \mathrm{C}$ model as regards the content of the token of 'boy' Jack uttered, we can discuss, relative to each of those options, the prospect of achieving a correct completion of the schema:

For any token $\tau$ of 'boy' and any property $\varphi, f(\tau)=\varphi$ iff

(1) [eligibility condition] ...;

(2) [selection condition] ....

What we will see is that it's over-determined that there can be no such correct completion. The first reason the schema can't have a correct completion is that none of (A)-(D) is true, and the second reason is that there can be no correct completion of the schema even given that one of (A)(D) is true. Let's see how this plays out with respect to each of (A)-(D).

$\operatorname{Re}(\mathbf{A})$ [Some precise property is the content of $\beta$ (the token of 'boy' Jack uttered)]. The token of 'Morgan is a boy' Jack uttered is vague. It's vague because even if it's determinately true or determinately false, it might have been neither; it might have been borderline true/false. The vagueness of the sentence token is entailed by the vagueness of $\beta$, the token of 'boy' contained in it, and, let's suppose, that is the only source of the sentence's vagueness. Given that and the assumption that Russellian propositions are the contents of declarative sentence tokens, the content of the sentence token Jack uttered is the precise proposition $\langle$ Morgan, $\varphi>$, if (A) is correct and $\varphi$ is the precise property that is the content of $\beta$. But how can the token of 'Morgan is a boy' be vague if its content is a precise proposition? That is possible if, and, I should think, only if, the epistemic theory of vagueness is correct. For that theory holds that a borderline sentence is true or else false, and we cannot know which. The fact that (A) presupposes the epistemic theory is already enough to make (A) problematic (see e.g. Schiffer 2003, pp. ...), but we can put that polemical remark aside, because, as we're about to see, we can show that (A) is false without having independently to show that the epistemic theory is false.

Penumbral shift entails that there is no such property as the property of being a boy. If there were such a property, it would be the content of every token of 'boy', and that is precisely what penumbral shift precludes. Given penumbral shift, if (A) is true, then different tokens of 'boy' may have different precise properties as their contents. In fact, the content of a particular token of 'boy' would have to be one of uncountably many precise properties that are equally eligible to be the content of a token of 'boy'. Let's ignore the fact that 'male' and 'human being' 
are vague and pretend that they express precise properties. Let's also pretend that for each person there is a precise instant of time at which he or she came into existence. Then a good candidate for being the content of $\beta$ if (A) is correct would be, say, the property of being a male human being whose age in seconds $\leq 531,066,240$. But if that is a good candidate, then so is the property of being a male human being whose age in seconds $\leq 531,066,240.1$, the property of being a male human being whose age in seconds $\leq 531,066,240.01$, the property of being a male human being whose age in seconds $\leq 531,066,240.001$, the property of being a male human being whose age in seconds $\leq 531,066,239$, the property of being a male human being whose age in seconds $\leq$ 531,066,239.05733, the property of being a male human being whose age in seconds $\leq$ 536,000,674, the property of being a male human being whose age in seconds $\leq 527,000,674.7009$, ...-well, you get the point. Let's suppose (for reductio) that (A) is true and that among the uncountably many equally good candidates for being the content of $\beta$ the one that happens to be its content is the property of being a male human being whose age in seconds $\leq 530,802,239.057$. Call this property $\varphi^{*}$. It can't be a brute inexplicable fact that $\varphi^{*}$ is $\beta$ 's content; there must be some feature that $\varphi^{*}$ alone among the countlessly many equally good candidates has which makes it the content of $\beta$. What might that feature be? It can't be that Jack intended to express $\varphi^{*}$ with $\beta .^{11}$ It can't be any causal relation that relates $\beta$ to $\varphi^{*}$ but to no other equally good candidate. And it's preposterous to suppose it's determined by the use of 'boy' among speakers of English. I submit that there is nothing that could make one alone of these countlessly many candidate properties the content of $\beta$, and that, therefore, (A) is false.

There is another, independent, reason why no precise property can be the content of $\beta$. If (A) were true, then, owing to penumbral shift, the meaning of 'boy' would have to be a function that was specifiable as an instance of the schema:

For any token $\tau$ of 'boy' and any precise property $\varphi, f(\tau)=\varphi$ iff

(1) [eligibility condition] ...;

(2) [selection condition] ....,

${ }^{11}$ For Jack to have such an intention he would have to have some way of thinking of the property under which he had that intention, but he clearly has no such way of thinking of it. See the discussion of de re intentions in Schiffer (2019). 
The considerations rehearsed in the preceding paragraph show that there can be no correct selection condition. But we can also see that there can be no correct eligibility condition. For let's continue to pretend that 'male' and 'human being' are precise and that for each person there is a precise instant of time at which he or she came into existence. Then in order for there to be a correct eligibility condition for 'boy' there would have to be real numbers $j, k$ such that (a) $j$ is the largest number such that, necessarily, every token of 'boy' is determinately true of every human male whose age in seconds $\leq j$ and (b) $k$ is the smallest number such that, necessarily, every token of 'boy' is determinately false of every human male whose age in seconds $\geq k$. If there were such numbers, then we could specify the eligibility condition by saying that:

For any token $\tau$ of 'boy' and any precise property $\varphi, f(\tau)=\varphi$ only if, for some real number $j^{\prime} \geq j,<k$, a male human being has $\varphi$ iff his age in seconds $\leq j^{\prime}$.

But there being such a $j^{\prime}$ is precluded by the fact that there can be no determinate cutoffs at any order of vagueness: owing to high-order vagueness, there can be no greatest lower bound and least upper bound to determine the precise properties that are eligible to be the content of a token of 'boy'.

(Some theorists, however, are evidently committed to rejecting the argument just concluded. The dominant model in formal semantics takes a compositional semantics for a language $L$ to be a compositional type-driven functional-application possible-worlds truththeoretic semantics for $L$ constructed in terms of the formal semanticist's proprietary language of the typed lambda calculus. This semantic model, like every other foundational semantic model, has been developed as an idealized model that prescinds from the vagueness of vague expressions, but some formal semanticists have attempted to show how at least certain kinds of vague expressions may be accommodated in a compositional semantics of the kind just mentioned. The most prominent such attempt is the semantics for vague gradable adjectives Christopher Kennedy (2007) offers. On that semantics, the meaning of 'tall' involves a contextually-determined measure function that maps individuals to precise degrees on a scale of measurement for height, so that an utterance of 'John is tall' in context $c$ is true if, for some precise degree of height $\delta$ onto which John is mapped by the measure function determined by $c$, John's degree of height $\geq \delta$, false otherwise. Since for Kennedy an utterance of 'John is tall' is either true or false, it's impossible for anyone to know the precise contextually-determined threshold John's height must exceed in 
order for the utterance to be true. So, as Kennedy recognizes, his account of vague gradable adjectives is an epistemic theory according to which, even though the context in which 'John is tall' is uttered determines a measure function which in turn determines a precise threshold that John's height must surpass in order for the sentence to be true relative to that context, it's impossible for anyone to know what that function or threshold is (for if one could know what the function was one could in principle know the threshold it determined, and if one could know that, then one could in principle determine whether the token of 'tall' produced in an utterance of 'John is tall' was true of any given individual, in which case nothing could be a borderline case of a thing of which the token was true, whereas the epistemicist, to account for what makes a predicate vague, must say that every utterance of a vague predicate admits the possibility of borderline cases). Even though it's impossible to know the measure function a context of utterance determines, we should still expect to be told something about how a context determines such a function. Yet all Kennedy tells us - by way of telling us how his theory provides a "contextualist" solution to the sorites paradox generated by a vague gradable adjective — is that if an utterance of 'tall' is being applied to a pair of individuals whose heights are very similar, then the context can't determine a measure function that determines a degree of height that only one of the pair of individuals meets or exceeds. In another much cited article, Chris Barker (2002) suggests that a dynamic component be added to the semantics of vague gradable adjectives, but his account, like Kennedy's, requires a contextually-determined precise threshold, and, like Kennedy's again, it offers no account of how that threshold is determined. ${ }^{12}$ )

Re (B) [No precise property is such that it's determinately the content of $\beta$, but myriad precise properties are each such that it's indeterminate whether it's the content of $\beta$.] Let $D$ be the determinately operator, where $D P$ means that it's determinately the case that $P$. As in effect already remarked (see n. 9), we don't want $P$ to entail $D P$ because we don't want $\neg D P$ to entail

\footnotetext{
${ }^{12}$ Una Stojnić and Matthew Stone (manuscript) claim to offer an account of how the Kennedyrequired precise cutoffs are determined. They propose that the work, or the better part of it, can be done by mechanisms of discourse coherence, but while they might show how discoursecoherence considerations affect the determination of thresholds, I don't see that they even attempt to show how Kennedy-required precise thresholds are determined.
} 
$\neg P$. Nevertheless, one can't be warranted in asserting $P$ unless one would be equally warranted in asserting that it was determinately the case that $P$-whence the infelicity of 'Morgan is a boy but it's indeterminate whether or not he's a boy'. Similarly, if one has the concept of a proposition's being determinately true and one knows that one knows that $p$ is true, then one knows that $p$ is determinately true, and if one is justified in believing that one is justified in believing that $p$ is true, then one is justified in believing that $p$ is determinately true. In the just concluded discussion of (A) I offered two arguments to show that every precise property is such that it couldn't be the content of $\beta$. I could not coherently have reached that conclusion and then added "But while the foregoing argument shows that every precise property is such that it couldn't be the content of $\beta$, it's still an open question whether or not there is a precise property such that it's indeterminate whether it's the content of $\beta$ '. This is because to whatever extent those arguments justify me in concluding that no precise proposition is the content of $\beta$, to that same extent they justify me in concluding that it's determinately the case that no precise proposition is the content of $\beta$ : Now, if it's determinately the case that no precise proposition is the content of $\beta$, then every precise property is such that it's determinately not the content of $\beta$; and if that is so, then no precise property is such that it's indeterminate whether it's the content of $\beta$. In short, the considerations that show (A) to be false also show (B) to be false.

It's also important to notice that the very same eligibility-condition problem that arises for (A) also arises for (B). This is because in order for a property to be such that it's indeterminate whether it's the content of a token of $\beta$, 'boy' must have a meaning that determines what must be the case in order for a property to be the content of a token of 'boy', and such a meaning, as we've seen, requires a correct eligibility condition for 'boy'. So the impossibility of such a condition falsifies (B) as well as (A).

$\operatorname{Re}(\mathbf{C})[$ Some vague property is the content of $\beta$ ]. If there are vague properties they are every bit as finely individuated as precise properties. So if a vague property $\varphi$ is a good candidate for being the content of $\beta$, then so are countlessly many vague properties that are virtually indistinguishable from $\varphi$. For example, suppose that vague properties $\varphi$ and $\psi$ are identical except that, whereas it is indeterminate whether $\varphi$ applies to a human male whose age in seconds is $522,000,000$, it is merely indeterminate whether it is indeterminate whether $\psi$ applies to him. Then we should expect 
that if either property is a good candidate for being the content of $\beta$, then so is the other. (A) was shown to be false by the fact that there was nothing to explain what would make any precise property such that it alone among the uncountably many equally eligible precise properties was the content of $\beta$. In the same way, $(\mathrm{C})$ is shown to be false by the fact that there is nothing to explain what would make any vague property such that it alone among the uncountably many equally eligible vague properties was the content of $\beta$.

Also like the falsity of (A), the falsity of (C) is overdetermined. If (C) were correct, there would be a correct completion of the M/C schema

For any token $\tau$ of 'boy' and any vague property $\varphi, f(\tau)=\varphi$ iff

(1) [eligibility condition] ...;

(2) [selection condition] ...,

but the argument of the preceding paragraph shows that there is no correct selection condition for this schema, just as the corresponding argument for precise properties showed that there could be no correct selection conditions for those properties. The discussion of (A) also contained an argument to show that there could be no correct eligibility condition for precise properties, and the same argument is available, mutatis mutandis, to show that there also can't be a correct eligibility condition for vague properties. If a vague property is the content of $\beta$ and Morgan has that property, then Jack's utterance of 'Morgan is a boy' is true, and if Morgan doesn't have that property, then the utterance is false, and if Morgan is a borderline instance of the property, then it's indeterminate whether the utterance is true. What matters as regards an eligibility condition are the conditions a property imposes on its determinate application. So, if we continue to pretend that 'male' and 'human being' are precise and that for each person there is a precise instant of time at which he or she came into existence, then, as before, in order for there to be a correct eligibility condition for the just-displayed schema there would have to be real numbers $j, k$ such that (a) $j$ is the largest number such that, necessarily, every token of 'boy' is determinately true of every human male whose age in seconds $\leq j$ and (b) $k$ is the smallest number such that, necessarily, every token of 'boy' is determinately false of every human male whose age in seconds $\geq k$. And if there were such numbers, then we could specify the eligibility condition by saying that:

For any token $\tau$ of 'boy' and any vague property $\varphi, f(\tau)=\varphi$ only if, for some real number $j^{\prime} \geq j,<k$, a human male has $\varphi$ iff his age in seconds $\leq j^{\prime}$. 
But there being such a $j^{\prime}$ is precluded by the fact that there can be no determinate cutoffs at any order of vagueness: owing to high-order vagueness there can be no greatest lower bound and least upper bound to determine even the vague properties that are eligible to be the content of a token of 'boy'. Therefore, every vague property, like every precise property, is such that it's not the content of $\beta$.

$\operatorname{Re}(D)$ [No vague property is such that it's determinately the content of $\beta$, but myriad vague properties are each such that it's indeterminate whether it's the content of $\beta$ ]. (D) stands to (C) as (B) stands to (A). I claim that the discussion of (C) warrants concluding that (C) is false, and to whatever extent it warrants holding that no vague property is the content of $\beta$, to that same extent it warrants holding that it's determinately the case that no vague property is the content of $\beta$, and that therefore each vague property is such that it's determinately not the content of $\beta$, and therefore no vague property is such that it's indeterminate whether it's the content of $\beta$. Just as the case against (A) is simultaneously a case against (B), so the case against (C) is simultaneously a case against (D).

\section{The Argument Extended}

The options (A)-(D) don't presuppose IBS. They are, I submit, the options available to any theorist who would claim that Russellian propositions are the contents of tokens of vague sentences. Consequently, if the arguments against (A)-(D) are sound, they show that Russellian propositions can't be the contents of tokens of vague sentences. And since the content of an unembedded sentence token is what a speaker means in producing that token, if what she means conforms to the meaning of the sentence uttered, those arguments also show that Russellian propositions aren't the things we mean. That isn't insignificant given the number of philosophers who claim that Russellian propositions are the contents of tokens of vague sentences. But would (A)-(D) fair better on another conception of propositions? When I provisionally supposed that we were addressing a view on which the contents of sentence tokens were taken to be Russellian propositions I implied that that was merely an expository convenience, since the arguments to be deployed to show that Russellian propositions couldn't be the contents of tokens of vague sentences could be redeployed to show that no propositions of any other kind could be their contents either. The idea is this. The arguments against (A)-(D) are effectually arguments against 
any theory that takes contents of tokens of vague predicates like 'boy' to be metaphysically-precise or metaphysically-vague properties. A theorist who takes the contents of sentence tokens to be propositions construed as functions from possible worlds to truth-values will construe properties as functions from possible worlds to sets of things in those worlds (e.g. she would represent the property of being a tiger as the smallest function that maps every possible world onto the set of things in that world that are tigers). It's not clear to me how the possible-worlds theorist might represent the notion of a metaphysically-vague property, but if we continue to pretend that 'male' and 'human being' are precise and that for each person there was a precise instant of time at which he or she came into existence, then the theorist would say that an example of a function eligible to be the content of $\beta$ (the token of 'boy' Jack uttered) might be, say, that function that maps each possible world onto the set of human males in that world whose age in seconds $\leq 530,156,794$. But if such a function is eligible to be the content of $\beta$, then so is the function that maps each possible world onto the set of human males in that world whose age in seconds $\leq 530,156,795$, the function that maps each possible world onto the set of human males in that world whose age in seconds $\leq 530,156,794.0057$, and so on for countlessly many other functions that differ only in some irrelevant way from them. But then the sort of considerations which show that there is nothing that could make one alone of the countlessly many equally eligible properties the content of $\beta$ would also show that there was nothing that could make one alone of the countlessly many equally eligible functions the content of $\beta$, and it should be obvious that the considerations pertaining to higher-order vagueness that made it impossible for there to be a correct eligibility condition for properties would make it equally impossible for there to be one for the functions that are the possible-world theorist's surrogates for properties. If anything is the content of $\beta$ - or if anything is such that it's indeterminate (or indeterminate whether it's indeterminate, or ...) whether it's the content of $\beta$ and it's not a property or property-surrogate, then it would have to be something that determines a property in the way that a Fregean "mode of presentation"/"way of thinking" of a property is supposed to do. But then there would be countlessly many equally eligible such things, and it should by now be obvious that they, too, would fail for the same sort of reasons that the other candidate contents fail.

From this I conclude that the contents of tokens of vague sentences aren't propositions of any kind. Further, if speaker-meaning were a relation, $S$ meant $p$, between a speaker $S$ and a proposition $p$ that she meant, then propositions would be the contents of tokens of vague sentences; 
so speaker-meaning isn't a relation between speakers and propositions (there is a freestanding argument for this in Schiffer 2019). But if propositions aren't the contents of sentence tokens, then what kind of things are there such that the meaning of an expression type can be a function that maps tokens of the expression onto those things? Nothing comes to my mind.

Davidsonians, however, claim that semantics has no need for such things as "contents," and that the only semantics a public language needs is a compositional truth theory, where a compositional truth theory for a language $L$ is a finitely statable theory of $L$ that generates for each truth-evaluable sentence of $L$ a theorem that specifies what must be the case in order for a token of that sentence to be true. Could such a semantics accommodate the vagueness of 'boy'? Some theorists would say the answer is yes. They would say that a compositional truth theory for English might have a base axiom that assigns Morgan to 'Morgan' and a base axiom for 'boy' to the effect that:

(1) For any person $x$, token $\tau$ of 'boy', and time $t, \tau$ is true of $x$ at $t$ iff $x$ is a boy at $t$,

so that the theory would then generate the theorem:

(2) For any token $\tau$ of 'Morgan is a boy', $\tau$ is true iff Morgan is a boy at the time $\tau$ was uttered.

Then they would say that this on its own already accommodates the vagueness of 'boy' in that its vagueness does nothing to disturb the truth of (1) or (2), and this, they would say, is because for each of (1) and (2) the vagueness of 'boy' on the right-hand side cancels out its vagueness on the left-hand side, in that each side matches the other "umbra for umbra and penumbra for penumbra" (Quine 1960, p. 37; quoted in Lepore and Ludwig 2005, p. 139). But this is wrong; there is no matching of sides umbra for umbra or penumbra for penumbra, and neither (1) nor (2) can be true. The considerations that defeat the proposition theorist's options (A)-(D) also defeat the Davidsonian's claim that natural languages admit of compositional truth theories. The problem is that the cancelling-out claim doesn't take account of penumbral shift. Owing to penumbral shift, different tokens of 'boy' may have somewhat different application conditions: one token may be true of a certain individual, another false of him, and a third neither determinately true nor determinately false of him. Now consider the token of (1) displayed above. It is true just in case every token of 'boy' has the same application conditions as the token of 'boy' on the right-hand side. But that entails that every token of 'boy' has the same application conditions as every other token of 'boy', and that is precisely what penumbral shift 
precludes. Penumbral shift entails that every token of (1) is false. The same argument, mutatis mutandis, also shows that every token of (2) is false.

What kind of base axiom for 'boy' should we expect a compositional truth-theoretic semantics to have for 'boy' in order for it to capture the context sensitivity induced by penumbral shift? Well, let's continue to pretend that 'male' and 'human being' are precise and that for each person there was a precise instant of time at which he or she came into existence. Let's now also pretend that there are real numbers $j, k$ such that $j$ is the largest number such that every token of 'boy' is determinately true of a male human being whose age in seconds $\leq j$ and $k$ is the smallest number such that every token of 'boy' is determinately false of every male human being whose age in seconds $\geq k$. Then we could devise a base axiom for 'boy' in a compositional truth theory provided we could find a condition $C$ such that:

For any $x$, token $\tau$ of 'boy', and time $t, \tau$ is true of $x$ at $t$ iff at $t x$ is a male human being whose age in seconds $=n$, for some real number $n$ such that:

(1) (eligibility condition) $j \geq n<k$;

(2) (selection condition) $n$ alone of the countlessly many eligible numbers - i.e. the uncountably many between $j$ and $k$-satisfies condition $C$.

But of course we already know that no such base axiom is to be found, for there can be no upper and lower bounds to serve as witnesses for ' $j$ ' and ' $k$ ' in the displayed conditions, and even if there were there would be no condition to do the job required for there to be a correct selection condition.

Might we, however, have a less demanding kind of compositional semantics, one that doesn't require there to be eligibility and selection conditions for vague predicates? After all, virtually every sentence we hear is vague, but we must know how they can be true, or false, since we have no trouble knowing many of them to be true, and some of them to be false. For example, you know that there are some people of whom every token of 'boy' must be true (e.g. your nine-year-old son Charlie), and some people of whom every token of 'boy' must be false (e.g. Ruth Bader Ginsburg), and this might seem to suggest that there are numerous conditions $C$ such that

(1) for any person $x$, token $\tau$ of 'boy', and time $t, \tau$ is true of $x$ at $t$ if $x$ satisfies $C$ at $t$

and numerous conditions $C^{*}$ such that

(2) for any person $x$, token $\tau$ of 'boy', and time $t, \tau$ is false of $x$ at $t$ if $x$ satisfies $C^{*}$ at $t$. 
Examples of such conditions might be, say:

(3) For any person $x$, token $\tau$ of 'boy', and time $t, \tau$ is true of $x$ at $t$ if at $t x$ is a male human being sixteen years old or younger.

(4) For any person $x$, token $\tau$ of 'boy', and time $t, \tau$ is false of $x$ at $t$ if at $t x$ is twenty-one years old or older.

And wouldn't that give us reason to think that 'boy' might be accommodated in some sort of at least partial compositional semantics in which such sentences, or ones like them, figured as base axioms? No, it wouldn't. For while every token of (3) and of (4) is determinately true, no token of (3) provides a true substitution instance of the open sentence (1) and no token of (4) provides a true substitution instance of the open sentence (2). To see this we need focus just on (1) and (3), since it will be obvious that what applies to them applies equally, mutatis mutandis, to (2) and (4). To say that there is a condition $C$ whose satisfaction is sufficient for such-and-such is for all that presently matters to say that having the property expressed by the open sentence ' $C(y)$ ' is sufficient for such-and-such. Consequently, (3) gives a true substitution instance of (1) only if the property expressed by the token in it of the open sentence

(5) At $t x$ is a male human being sixteen years old or younger expresses a property possession of which by an individual is sufficient for a token of 'boy' being true of him (provided the token is produced while he has the property). But if any token of (5) expressed a property, that property would be its content. Yet (5) is a vague predicate that would be taken to be context insensitive if its vagueness were being ignored (see the discussion of predicates* on p. 000), and if the arguments of $\S I I I$ are sound, they show that no property can even be such that it's indeterminate whether it's the content of such a predicate. All this is entirely consistent with every token of (3) being determinately true. As I said when I introduced the notion of penumbral shift, the penumbras of tokens of a vague expression could vary only so much. For most predicates, there will always be certain things to which every token of the predicate applies, and certain things to which they don't apply. For example, although 'wealthy' is very vague, every token of 'Jeff Bezos is wealthy in 2019' is determinately true. In the same way, every token of 'boy' will be determinately true of a person if any token of (5) is true of him.

It would appear that, if everything I've said up to this point is true (a big if, to be sure), then no spoken language enjoys anything worth calling a compositional truth-theoretic semantics 
(henceforth, a compositional semantics, for short).

\section{What Now?}

Some theorists evidently take it to be a datum that every spoken language has a compositional semantics. But it's not a datum. To be justified in claiming that every spoken language has a compositional semantics, one would have to be justified in claiming that there is some feature of every spoken language that couldn't be explained unless the language had a compositional semantics. What might that feature be? Davidson (2001) said that we wouldn't be able to learn a language if it didn't have a compositional semantics, and this thought gets re-expressed in the claim that we need to suppose that a language has a compositional semantics in order to explain how we are able to understand novel sentences of the language. Usually, when these claims are dissected, the appeal to understanding is something of a red herring, in that the point really being relied on is that a compositional semantics for a language is needed to explain how each of its infinitely many expressions gets its meaning, or how infinitely many sentences get their truth conditions. One often hears it said, for example, that a compositional semantics is needed to explain the fact that there are infinitely many true instances of the schema

' $S$ ' is true iff $S$,

such as that old chestnut

'Snow is white' is true iff snow is white.

But I would say that, since 'snow' and 'white' are vague, penumbral shift renders 'Snow is white' context sensitive in a way that makes every token of the just displayed T-sentence false, and that the same goes for virtually every sentence we hear, since virtually every one of them is vague too.

Still, indefinitely many utterances of vague sentences are known to be determinately true and just as many are at least capable of being known to be determinately false. We have a decent idea of how this might be explained if we can assume that our language has a compositional truththeoretic semantics. How might it be explained on the assumption that our language doesn't have a compositional semantics? I will close by sketching a rough idea of how such an explanation might be possible. The sketch moves the spotlight away from public language and onto the system of mental representation in which we process information.

So suppose we think, i.e. process information, in a neural system of mental representation $M$. Here, to set up a foil, is in broad outline the sort of theory of $M$ one might have heard from a 
proponent of intention-based semantics back in the day who, as he always did, was ignoring vagueness and aiming to show that mentalese sentences are "the basic objects of interpretation; their content confers content upon thoughts; and thoughts transmit their content to outer speech" (McGinn 1982, p. 70). Versions of the line, which I'll call the two-component story, may be found in Field (1977, 1978), Loar (1981, 1982), and Schiffer (1981).

\section{The Two-Component Story}

The meaning of an $M$ expression has two components: a conceptual role component and a truththeoretic component. The two components are conceptually distinct, and neither determines the other. Conceptual role is a generalization of functional role. Functional roles are properties of states defined by ways the state is counterfactually related to sensory stimulation, to other states, and to bodily movement. There is a certain functional role whose possession by a state constitutes its being a belief, another functional role whose possession by a state constitutes its being a desire, and so on. Two states that are beliefs will share the conceptual role that makes them both beliefs, but if one is, say, a belief that moths have souls and another is a belief that ontogeny recapitulates phylogeny, the total functional roles of the states will be very different. Propositional-attitude states are realized by tokenings of $M$ sentences. If the $M$ correlate of 'Elvis is alive' is tokened in a state with the belief-making functional role, then one believes that Elvis is alive; if it's tokened in a state with the desire-making functional role, then one desires that Elvis is alive. The conceptual role of a sentence tokened as a belief determines that functional role of the resultant belief state which distinguishes its functional role from those of one's other belief states. The conceptual role of every complex expression is determined by the conceptual roles of the sentence's constituent words and syntactic structures, but a conceptual role for an individual word can in most cases only be determined by an enormously complex functional theory that determines the term's conceptual role in tandem with every other word's conceptual role.

There is a compositional truth theory for $M$, and the truth-theoretic component of an $M$ expression's meaning is simply the semantic value assigned to the expression by that truth theory. The truth-theoretic properties of a spoken language expression are, and are determined by, those of its $M$ correlate, so our spoken language has a compositional truth-theoretic semantics that derives from the compositional truth-theoretic semantics for $M$. The Gricean program of intentionbased semantics is supposed to tell us how a population must use a language $L$, construed as a 
pairing of sounds and semantic values, so that each expression in $L$ has in each member of the population an $M$ correlate whose semantic properties match its semantic properties. Understanding a foreign language consists, in effect, of having tacit knowledge of the compositional semantics that the language inherits from its speakers' mentalese. Explaining how you are able to know the truth-values of utterances of novel sentences is now a snap: a compositional semantics for our public language derives from the compositional semantics for $M$; you have tacit knowledge of that semantics; thanks to that tacit knowledge you know that so-and-so's utterance of a certain sentence is true iff such-and-such is the case; and thanks to the belief-forming mechanisms that determine how you process information in $M$, you know that such-and-such is the case, and thereby know that the utterance is true.

Two-component theorists were aware of a soft spot in the truth-theoretic component of their theory. The base axioms of the compositional truth-theory for $M$ assign referential semantic values to words in M's lexicon, and those assignments must be correct if the compositional truth theory is to be correct. There are reference relations that determine whether or not these assignments are correct: an assignment of semantic value $v$ to a word $w$ is correct only if an appropriate reference relation correlates $v$ with $w$. It's very important to the success of the twocomponent strategy that there are physicalistically respectable explications of these reference relations. Initially it was hoped that these explications could be given in terms of the head-world causal relations that account for the reliability of the information processing through which our beliefs are formed - for example, relations that would explain why your believing that you are in the shower is extremely good evidence that you are indeed in the shower (see Field 1972). But the best efforts to find these explications resulted in nothing but frustration, and leading twocomponent theorists began to wonder whether there was any theoretical need for these elusive reference relations after all, and this in turn led them to explore "deflationary" theories of meaning and content in which there is no need for reference relations and truth conditions to play any explanatory role, and therefore no need for them whatever (see e.g. Leeds 1978; Field 1994; Schiffer 1981, 1987). Erstwhile two-component theorists ought to be sympathetic to the contrasting line on mentalese that I will now briefly sketch.

\section{The One-Component Story}

The two-component story is one that can be accepted only by a theorist who is making no effort 
to accommodate the vagueness of vague expressions. If the theorist were to attempt to explain the meaning of vague expressions without pretending they weren't vague, she ought, I believe, to discover that vagueness is the biggest soft spot in her theory. The one-component story is a line of thought that aims to explain what needs to be explained without running afoul of the now familiar traps set by vagueness. In the one-component story about meaning in $M$, expressions of $M$ have compositionally-determined conceptual roles, but there is no compositional truth-theoretic semantics for $M$. Of course, we do use semantic terms, so the $M$ correlates of these terms will be in $M$ 's lexicon. For example, if $M$ is a neural version of English, then tokened in one's belief-box might be sentences such as:

Jill's utterance of 'Jack is feckless' is true.

and

In uttering 'He's feckless' Jill was referring to Jack.

And it might be that the conceptual role of 'true' in $M$ is such that, for any sentence $\sigma$,

$\sigma$

is tokened in one's belief-box when, and only when,

${ }^{\top} \sigma^{\top}$ is true

and

'It's true that $\sigma^{\prime}$

are also tokened there.

The vagueness of predicates* gets handled in the following way ${ }^{13}$ when we take what applies to 'boy' in $M$ to apply to all predicates* in $M$. A constant feature of the conceptual role of 'boy' is that there are certain predicates $\Psi$ and singular terms $\alpha$ such that, if

${ }^{\top} \alpha$ is $\Psi^{1}$

${ }^{13}$ A predicate*, you may recall (see p. ...), is a vague predicate that would be regarded as having a context-insensitive meaning if it's vagueness were ignored. The treatment of all vague terms is similar; I'm confining myself to predicates* only because explaining how penumbral shift works for terms - e.g. 'tall' - that are taken to be context-sensitive even when their vagueness is ignored would direct us away from the important points that need to be emphasized. 
is tokened in one's belief-box then

${ }^{\top} \alpha$ is a boy'

is also tokened there. ${ }^{14}$ For example, if

Morgan is a nine-year-old human male

is tokened in one's belief-box, then

Morgan is a boy

will also be tokened there, as will

It's true that Morgan is a boy

and

I believe that Morgan is a boy,

and if

Jane uttered 'Morgan is a boy'

is also tokened there, then so will be

Jane's utterance is true.

But there isn't a determinate set of predicates whose tokening would automatically secure the concurrent tokening of 'boy' in the way indicated, and there are often interpersonal differences in the word's conceptual role so that, whereas a tokening

${ }^{\ulcorner} \alpha$ is $\Psi^{1}$

in Jill's belief-box would automatically cause a belief-box tokening of

' $\alpha$ is a boy',

it wouldn't have the same effect for Jack. There are, however, always predicates - 'nine-year-old human male' being one - that must always secure belief-box tokenings of 'boy' for a person on pain of otherwise leading others to judge that she doesn't mean by 'boy' what they mean by it. Penumbral shift is a nonpermanent change in conceptual role that can occur for various reasonse.g. because of what David Lewis (1979) called accommodation, because of what at the moment is at stake in how you use a particular vague expression, or, more often than not, because of no discernible rhyme or reason.

On the two-component story, understanding one's own or any other spoken language requires there to be a compositional semantics for the language of which one has tacit knowledge.

${ }^{14}$ I'll leave it to the reader to read in the requisite ceteris paribus clauses. 
On the one-component story neither a spoken nor a mentalese language has a compositional semantics, and there is nothing more to "understanding" one's own language than its expressions' having correlates in $M$. Understanding a foreign language, however, requires a pairing of the foreign expressions with one's own on the basis of relevant similarities of conceptual roles, where what counts as a relevant similarity is some function, to be decided on pragmatic grounds, of the pairing's ability to enable one to explain and predict the behavior of the foreign speakers and to use their utterances as a source of information about the external world (cf. Field ...). Suppose we arrived at an acceptable "translation" of foreign language $L$ into our language based on what we took to be the conceptual roles of the mentalese correlates of $L$ 's expressions. Then on the basis of our beliefs about how speakers of $L$ formed their beliefs and intentions, we could form beliefs about what they believed and intended, so that sentences like 'Foreigner $\mathrm{X}$ wants to eat at that restaurant' would get tokened in our belief-boxes. We might also accept what we took to be an explanation of how foreigner $X$ is able to know that an utterance of a novel sentence of her language is true. We may know that she understands $L$ because we know she thinks in mentalese correlates of $L$, and we know enough about the conceptual roles of those correlates to know the beliefforming procedures by virtue of which, for some $L$ sentence $\sigma$, tokened in her belief-box are the mentalese correlates of her spoken sentences that we would "translate" as:

- 'In uttering ' $\sigma^{\top} Z$ said that $\sigma^{\top}$

- $\sigma$

- $Z$ 's utterance of ' $\sigma$ ' is true

And since we "translate" $\sigma$ into our language as 'Newton did alchemical experiments', and since that sentence is tokened in our belief-boxes, it might easily be that also tokened in our belief-boxes are

- $X$ knows that in uttering ${ }^{\top} \sigma^{\top} Z$ said that Newton did alchemical experiments

- $X$ knows that Newton did alchemical experiments

- $X$ knows that $Z$ 's of ' $\sigma$ is true

Does this count as a correct explanation of how a speaker is able to know that the utterance of a novel sentence of her language is true? It would, I think, if you suppose yourself to be living in the world the one-component story describes. And if you question whether it really explains how we can know that an utterance of a novel sentence is true, then you're raising that question 
from the perspective of an alien with knowledge of the conceptual roles of some creatures' neural sentences which you haven't yet succeeded in pairing with your own sentences. Once you achieve a pairing, you will make a different judgment.

I wish I could discuss these difficult issues with Brian. ${ }^{15}$

${ }^{15}$ Discussions with Crispin Wright on the topics and views of this paper were very helpful. Some of this material was presented in the NYU Mind and Language Seminar that Cian Dorr and Jim Pryor gave in spring 2018. I benefited from their comments and from the comments made by Chris Barker and others during the general discussion. 


\section{Works Cited}

Almog, J., Perry, J, and Wettstein, H. (eds). (1989). Themes from Kaplan (Oxford University Press).

Barker, C. (2002). “The Dynamics of Vagueness," Linguistics and Philosophy, 25 (1): 1-36.

Capone, A. (ed). (2019). Further Advances in Pragmatics and Philosophy, Part II (Springer).

Davidson, D. (2001). "Theories of Meaning and Learnable Languages," in Inquiries into Truth and Interpretation (Oxford University Press): 3-15.

Evans, G. and McDowell, J. (eds.) (1976). Truth and Meaning: Essays in Semantics (Oxford University Press).

Field, H. (1972). “Tarski's Theory of Truth,” Journal of Philosophy, 69: 347-375.

(1977). “Logic, Meaning, and Conceptual role,” Journal of Philosophy, 74: 379-409.

(1978). "Mental Representation,” Erkenntnis, 13: 9-61.

(1994). "Deflationist Views of Meaning and Content," Mind, 103: 249-285.

Grice, H. P. (1957). “Meaning,” Philosophical Review, 66: 377-88.

(1969). “Utterer's Meaning and Intentions," Philosophical Review, 78: 147-77.

Gunderson, K. (ed.). (1975). Minnesota Studies in the Philosophy of Science (University of Minnesota Press).

Hale, R., Wright, C., and Miller, A. (eds.). (2017). Blackwell Companion to the Philosophy of Language, Volume 1, Second Edition (Wiley-Blackwell).

Kaplan, D. (1989a). "Demonstratives: An Essay on the Semantics, Logic, Metaphysics, and Epistemology of Demonstratives and Other Indexicals,” in Almog et. al. (1989). (1989b). "Afterthoughts," in Almog et. al. (1989).

Kennedy, C. (2007). "Vagueness and grammar: The semantics of relative and absolute gradable adjectives," Linguistics and Philosophy 30: 1-45.

Leeds, S. (1978). “Theories of Reference and Truth,” Erkenntnis, 13: 111-129.

Lepore, E. and Ludwig, K. (2005). Donald Davidson: Meaning, Truth, Language, and Reality (Oxford University Press).

Lewis, D. (1969). Convention: A Philosophical Study (Harvard University Press). (1975). “Languages and Language,” in Gunderson (1975): 3-35. (1979). "Scorekeeping in a Language Game," Journal of Philosophical Logic 8(1): 339- 
59.

Loar, B. (1975). “Two Theories of Meaning” in Evans and McDowell (1975).

(1981). Mind and Meaning (Cambridge University Press).

(1982). “Conceptual Role and Truth Conditions," Notre Dame Journal of Formal

Logic, 23: 272-83.

Neale, S. (2016). "Silent Reference,” in Ostertag (2016): 229-342.

Quine, W. V. O. (1960). Word and Object (MIT Press).

Ostertag, G. (ed.) (2016). Meanings and Other Things: Themes from the Work of Stephen Schiffer (Oxford University Press).

Schiffer, S. (1972). Meaning (Oxford University Press).

(1981). "Indexicals and the Theory of Reference," Synthese, 49: 43-100.

(1987). Remnants of Meaning (MIT Press).

(2003). The Things We Mean (Oxford University Press).

(2016). "Reply to Neale," in Ostertag (2016): 495-501.

(2017). "Intention and Convention in the Theory of Meaning," in Hale et. al. (2017):

49-72.

(2019). "Vague Speaker-Meaning" in Capone (2019).

Stojnić, U. and Stone, M. (manuscript). "Vague Utterances in Context." 